Case Report Journal of Epilepsy Research pISSN 2233-6249 / elSSN 2233-6257

Received October 27, 2017

Accepted November 23, 2017

Corresponding author: Dae Won Seo

Department of Neurology, Samsung

Medical Center, Sungkyunkwan

University School of Medicine, 81

Irwon-ro, Gangnam-gu, Seoul 06351,

Korea

Tel. +82-2-3410-3595

Fax. +82-2-3410-0052

E-mail; daewon@skku.edu

\title{
Intractable Epilepsy with Solitary Cerebral Calcification
}

\author{
Kyoung Jin Hwang ${ }^{1}$, Jiyoung Kim², Eun Yeon Joo ${ }^{3}$, Seung Bong Hong ${ }^{3}$, \\ Seung-Chyul Hong ${ }^{4}$, Dae Won $\mathrm{Seo}^{3}$
}

${ }^{1}$ Department of Neurology, Kyung Hee University School of Medicine, Seoul; ${ }^{2}$ Department of Neurology, Busan Medical Center, Busan; ${ }^{3}$ Department of Neurology, Samsung Medical Center, Sungkyunkwan University School of Medicine, Seoul; ${ }^{4}$ Department of Neurosurgery, Samsung Medical Center, Sungkyunkwan University School of Medicine, Seoul, Korea

\begin{abstract}
Cerebral calcification is a common incidental finding upon brain imaging and its epileptogenicity is often underestimated. Here, we report a case of intractable epilepsy arising in conjunction with a solitary cerebral calcification. A 42-year-old male with intractable epilepsy was admitted to the epilepsy clinic for invasive epilepsy surgery. Brain magnetic resonance imaging revealed a slight high-intensity signal change in the right amygdala and a small, calcified lesion in the right lateral temporal region. The patient underwent invasive monitoring with subdural electrodes. He had five habitual seizures with automatisms and fast activity. These seizures initiated in the right lateral temporal area just above the solitary calcified lesion. Neuropathology of the calcified lesion showed no specific findings apart from a fibrocalcific nodule. Thus, although solitary cerebral calcifications may be an asymptomatic or coincidental finding in some patients, they may also have a highly epileptogenic focus. (2017;7:126-128)
\end{abstract}

Key words: Intractable epilepsy, Cerebral calcification, Epilepsy surgery

\section{Introduction}

The development of brain imaging techniques for evaluating patients with intractable epilepsy has allowed for the identification of various structural brain lesions that may function as epileptogenic foci, such as those due to cortical dysplasia, vascular malformations, or tumors. Cerebral calcifications are a common incidental finding, although the epileptogenicity of these structures is often underestimated. Cerebral calcifications arise from the deposition of crystalline calcium in the brain parenchyma due to either physiological or pathological conditions, such as infection or metabolic, neoplastic, vascular, congenital, developmental, or traumatic causes. Here, we report a case of intractable epilepsy arising from a solitary cerebral calcification.

\section{Case}

A 42-year-old, right-handed male was admitted to the epilepsy clinic with a history of drug-resistant seizures since the age of 18 . His frequent seizures included psychic auras that often progressed to the loss of awareness and clonic movement of his left arm and leg. These episodes occurred while taking carbamazepine, valproate, and levetiracetam.

He had no previous medical history of trauma, no history of eating raw fish or meat, and no pertinent family history. His neurological examination and laboratory studies, including eosinophil count and calcium levels, were normal. Brain magnetic resonance imaging revealed a slightly high signal change in the right amygdala and a small, calcified lesion $(4.5 \times 4.7 \mathrm{~mm})$ in the right lateral temporal region (Fig. 1). A video electroencephalography (EEG) monitoring study demonstrated frequent interictal epileptiform activity localized
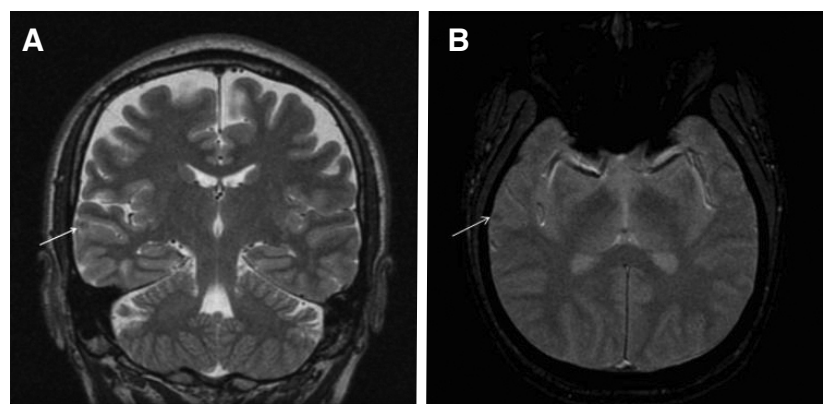

Figure 1. Brain magnetic resonance imaging showing a solitary cerebral calcification (A) T2-weighted coronal view, (B) gradient echo axial view. 
in the right temporal region. Ictal EEG exhibited maximum amplitude over the right frontotemporal electrodes and ictal single-photon emission computed tomography (SPECT) showed hyperperfusion in the right anterior and mesial temporal regions. Brain 18 F-fludeoxyglucose positron emission tomography (FDG-PET) revealed hypometabolism in the right temporal region, including the mesial temporal area. A multidisciplinary epilepsy case review panel decided to perform invasive monitoring with subdural electrodes.

A right-sided craniotomy was performed to place a subdural electrode grid, as well as strip electrodes, over the basal, mesial, and lateral temporal regions. Platinum contact electrodes embedded in 0.5-mm-thick flexible silicone plates (AD-Tech Medical Instrument Corp, Racine, WI, USA) were used. A 64-channel video EEG recording device was used to obtain the measurements. Four days after electrode placement, the patient had five habitual seizures with automatisms featuring right hand fumbling but no aura. During these seizures, fast activity was observed to initiate in the right lateral temporal area just above the solitary calcified lesion (Fig. 2B, C: electrode $\mathrm{H} 15)$. From that point, activity spread into the basal region and then the entire lateral temporal region (Fig. 2). Five days after electrode placement, subclinical seizures initiating above the mesiobasal temporal lesion and spreading over the temporal region were detected via EEG. Six days after electrode placement, the patient underwent
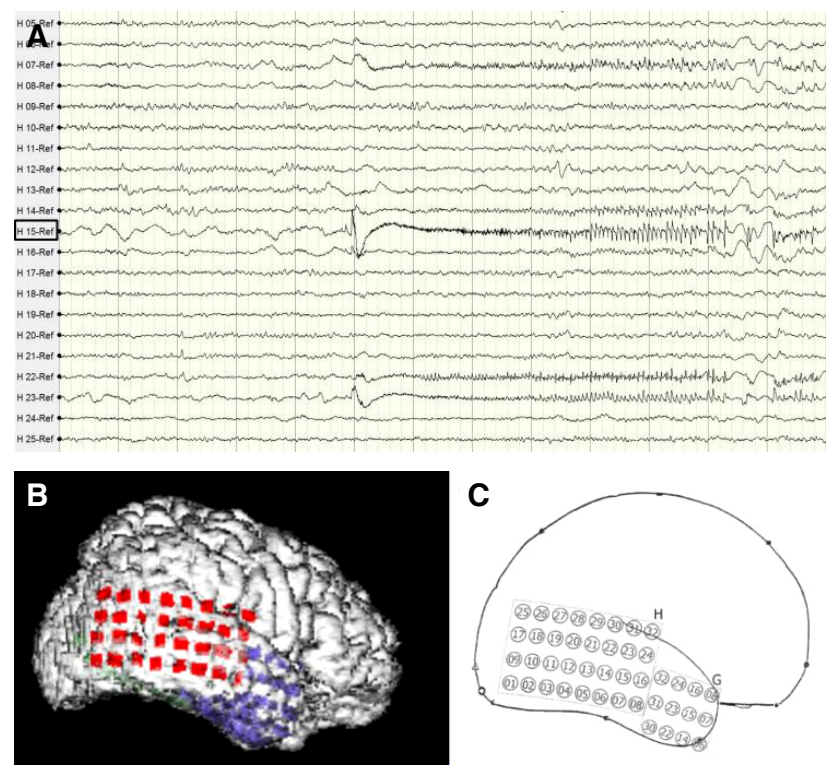

Figure 2. (A) Subdural electroencephalography showing fast activity initiating at the electrode above the solitary cerebral calcified lesion $(\mathrm{H}-15)$ during a habitual seizure. (B) and (C) show the locations of the subdural electrodes. an anterior temporal lobectomy with partial amygdalohippocampectomy. Histological examination of the small, calcified lesion showed no specific findings apart from being a fibrocalcific nodule (Fig. 3). Examination of the amygdala showed deposits of corpora amylacea, but the hippocampus showed no abnormalities. The patient experienced no further events and has been seizure-free for 24 months without antiepileptic drugs.

\section{Discussion}

This report describes a patient with intractable epilepsy arising from a solitary cerebral calcification. Unfortunately, it was not possible to determine the etiology of this lesion, as the neuropathological findings were inconclusive apart from the presence of a fibrocalcific nodule.

The identification of focal lesions is particularly important when patients with medically refractory partial seizures are considered for epilepsy surgery. Indeed, the best outcomes after surgery are observed when focal structural lesions are identified and are concordant with EEG findings. In a previous neuropathological report, the most common causes of intractable epilepsy were focal cortical dysplasia, scar lesions due to hypoxia/ischemia or trauma, and brain tumors. Cerebral infections such as neurocysticercosis and tuberculous meningitis were not commonly observed, except in endemic

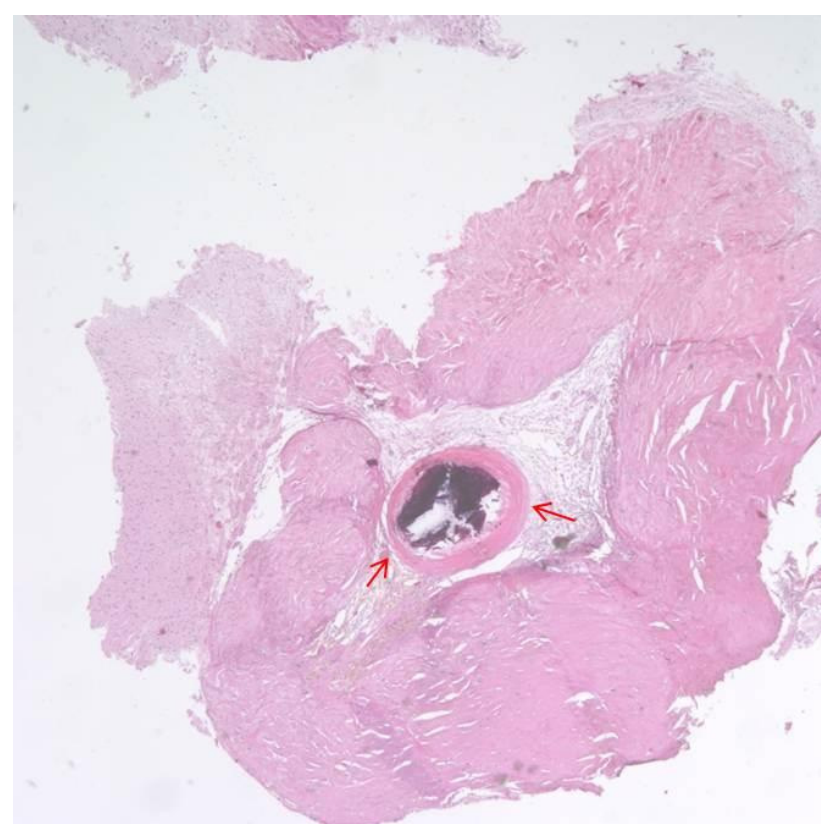

Figure 3. Pathological findings of the resected right lateral temporal cortex (hematoxylin-eosin staining; original magnification, $\times 40$ ). A calcified nodule (arrows) can be observed surrounded by fibrotic tissue. 
areas. ${ }^{1}$ Single-lesion neurocysticercosis might cause seizure recurrence in $14.5 \%$ of patients. ${ }^{2}$

A solitary cerebral calcification is defined as a single small $(<10$ $\mathrm{mm}$ ) calcified lesion. ${ }^{3}$ The most common causes of these lesions are cerebral infections or their sequelae, including cysticercosis, tuberculomas, or fungal granulomas. Other causes of solitary cerebral calcifications include low-grade calcifying tumors, such as oligodendrogliomas, gangliogliomas, or dysembryoplastic neuroepithelial tumors, ${ }^{4}$ and vascular lesions such as small arteriovenous malformations. ${ }^{3,5,6}$ In this case, a neuropathological analysis of the lesion revealed no other primary disease.

Patients with solitary cerebral calcification-related epilepsy may show varying courses, from simple to intractable seizures. In a previous imaging study ${ }_{1}^{7,8}$ gliosis or edematous changes around a cerebral calcified lesion and persistent enhancement of the lesion predicted poor seizure control. Poor seizure control was due to the development of perilesional pathognomonic changes due to inflammatory aggravation caused by an underlying disease $e^{9}$ or recurrent seizures. EEG abnormalities, a family history of epilepsy, and serial seizures were found to be additional risk factors for seizure recurrence. $^{9}$

In this case, the patient showed a subclinical seizure arising from the mesiobasal temporal region. Brain SPECT and FDG-PET also suggested defects in the mesiobasal temporal area, and neuropathological assessment of the amygdala revealed deposits of corpora amylacea. Epilepsy due to solitary cerebral calcification may arise not only from the calcification itself but also from the adjacent mesial temporal cortex, perhaps representing a secondary kindling phenomenon. In a previous study examining the co-occurrence of calcified cysticerci and hippocampal sclerosis, about $8 \%$ of calcifications were located in the temporal lobe, ipsilateral to the hippocampal sclerosis. ${ }^{10,11}$

Thus, although solitary cerebral calcifications may be an asymptomatic or coincidental finding in some patients, they may also function as an important epileptogenic focus. Therefore, focal cortical calcification should be carefully observed in patients with intractable epilepsy.

\section{References}

1. Piao YS, Lu DH, Chen $L$, et al. Neuropathological findings in intractable epilepsy: 435 Chinese cases. Brain Pathol 2010;20:902-8.

2. Singhi $P$, Suthar $R$, Deo $B$, Malhi $P$, Khandelwal NK. Long-term clinical and radiologic outcome in 500 children with parenchymal neurocysticercosis. Pediatr Infect Dis J 2017;36:549-55.

3. Singh G, Sachdev MS, Tirath A, Gupta AK, Avasthi G. Focal cortical-subcortical calcifications (FCSCs) and epilepsy in the Indian subcontinent. Epilepsia 2000;41:718-26.

4. Fried I, Kim JH, Spencer DD. Limbic and neocortical gliomas associated with intractable seizures: a distinct clinicopathological group. Neurosurgery 1994;34:815-23; discussion 823-4.

5. Pal DK, Carpio A, Sander JW. Neurocysticercosis and epilepsy in developing countries. J Neurol Neurosurg Psychiatry 2000;68:137-43.

6. Murthy JM, Subba Reddy YV. Prognosis of epilepsy associated with single CT enhancing lesion: a long term follow up study. J Neuro/ Sci 1998;159:151-5.

7. Agarwal A, Raghav S, Husain M, Kumar R, Gupta RK. Epilepsy with focal cerebral calcification: role of magnetization transfer MR imaging. Neurol India 2004;52:197-9.

8. Nash TE, Pretell J, Garcia HH. Calcified cysticerci provoke perilesional edema and seizures. Clin Infect Dis 2001;33:1649-53.

9. Sharma LN, Garg RK, Verma R, Singh MK, Malhotra HS. Seizure recurrence in patients with solitary cystic granuloma or single parenchymal cerebral calcification: a comparative evaluation. Seizure 2013; 22:840-5.

10. Leite JP, Terra-Bustamante VC, Fernandes RM, et al. Calcified neurocysticercotic lesions and postsurgery seizure control in temporal lobe epilepsy. Neurology 2000;55:1485-91.

11. Rathore C, Thomas B, Kesavadas C, Radhakrishnan K. Calcified neurocysticercosis lesions and hippocampal sclerosis: potential dual pathology? Epilepsia 2012;53:e60-2. 